\title{
Microencapsulation of fish oil-in-water emulsion using thiol-modified $\beta$-lactoglobulin fibrils-chitosan complex
}

\begin{abstract}
Fish oil was encapsulated via spray-drying using different $\beta$-lactoglobulin ( $\beta$-LG) fibril variants, chitosan and maltodextrin. The effects of different wall materials and inlet temperatures on the physicochemical properties of fish oil microcapsules were investigated. A lower polydispersity index (0.29-0.37) coupled with larger mean droplet size $(0.96-1.25 \mu \mathrm{m})$ and higher zeta potential $(41.3-41.6 \mathrm{mV})$ were achieved in the reconstituted fish oil microcapsules formed using thiolmodified $\beta$-LG fibril/ $0.5 \%$ chitosan complex. Lower angle of repose $\left(<30^{\circ}\right)$ displayed by the same sample suggested good flow properties, and this finding correlated well with its smooth surface, as observed using scanning electron microscopy. Microcapsules stabilized using the complex exhibited comparable encapsulation efficiency and slightly higher glass transition temperature than that of using unmodified $\beta$-LG fibrils, indicating the former's greater heat stability. This study provided valuable insight into the application of protein fibrils-polysaccharide complexes as an effective encapsulation agent.
\end{abstract}

Keyword: Microcapsules; Biopolymer complex; Spray drying; Morphology; Encapsulation efficiency; Reconstitution properties 